\title{
I-Gel is a suitable alternative to endotracheal tubes in the laparoscopic pneumoperitoneum and trendelenburg position
}

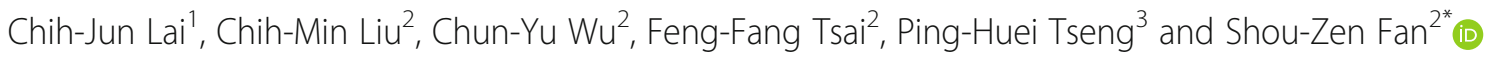

\begin{abstract}
Background: The use of supraglottic airway devices (SADs) in surgeries with laparoscopic pneumoperitoneum and Trendelenburg (LPT) positioning is controversial due to concerns about insufficient pulmonary ventilation and aspiration. In this prospective, randomized-controlled trial, we evaluated whether the i-gel, a new second generation $S A D$, provides an effective alternative to an endotracheal tube (ETT) by comparing respiratory parameters and perioperative respiratory complications in non-obese patients.
\end{abstract}

Methods: In a randomized controlled trial, forty anesthetized patients with ASA I-II were divided into equally sized i-gel and ETT groups. We evaluated the respiratory parameters in the supine and LPT position in comparison between the two groups. The leak fraction was our primary outcome, which was defined as the leak volume divided by the inspired tidal volume. The leak volume was the difference between the inspired and expired tidal volumes. We also monitored pulmonary aspiration and respiratory complications during the perioperative period.

Results: In the LPT position, there were no differences in the leak fraction (median [IQR]) between the i-gel and ETT groups (6.20[3.49] vs 6.38[3.71] \%, $P=0.883$ ). In the i-gel group, notably less leakage was observed in the LPT position than in the supine position (median [IQR]: $7.01[3.73] \%$ ). This phenomenon was not observed in the ETT group. The rate of postoperative sore throat was also significantly lower in the i-gel group than in the EIT group (3/17 vs 9/11). No vomitus nor any signs associated with aspiration were noted in our patients after extubation in the follow-up prior to discharge.

Conclusions: The i-gel provides a suitable alternative to an ETT for surgeries with LPT positioning in non-obese patients.

Trial registration: Registered at Clinicaltrials.gov NCT02462915, registered on 1 June 2015.

Keywords: Endotracheal tube, i-gel, Laparoscopic surgery, Leak fraction, Pneumoperitoneum, Respiratory parameters, Trendelenburg position

\section{Background}

Surgeries that require laparoscopic pneumoperitoneum and Trendelenburg (LPT) positioning are becoming popular. However, this positioning causes a cephalic shifting of viscera and diaphragm. The changes in respiratory mechanics following patient receiving LPT position, may

\footnotetext{
* Correspondence: shouzen@gmail.com

This report has been submitted in part, to the 2016 Euroanaesthesia. ${ }^{2}$ Department of Anesthesiology, National Taiwan University Hospital, No. 7, Zhung Shan S. Rd., Zhongzheng Dist., Taipei City 10002, Taiwan (R.O.C.) Full list of author information is available at the end of the article
}

result in increased airway pressure [1]. It has also been associated with potential increases in episodes of gastric regurgitation $[2,3]$. Tracheal intubation to secure airway is the gold standard in surgeries requiring LPT positioning. Recently, trends in airway management have progressed from using an endotracheal tube (ETT) to a supraglottic airway device (SAD) because of the advantages that such devices confer [4-6]. However, the use of SADs in surgeries requiring LPT positioning remains controversial because of the increased risk of insufficient ventilation and pulmonary aspiration [7-9]. 
The second-generation SADs with gastric channel provide higher sealing pressures and more complete airway protection than the laryngeal mask airway classic [10-13]. The i-gel (Intersurgical, Wokingham, UK) is a new secondgeneration $\mathrm{SAD}$. It includes the non-inflatable cuff, and a buccal stabilizer to prevent malposition [14]. It provides lower respiratory complications and is capable of sealing higher oropharyngeal leak pressures than earlier SADs [15]. The device is fabricated from styrene ethylene butadiene styrene (SEBS) [16], and provides improved sealing pressure when warming up to body temperature [17]. Although the igel has proven effective and safe for use in elective surgeries using positive ventilation, there is limited evidence to support the use of an i-gel in surgeries requiring LPT positioning.

In this study, we predicted that i-gel would perform comparably compared with an ETT in the LPT position by comparing respiratory parameters and perioperative complications in non-obese patients.

\section{Methods}

\section{Patients and protocol}

A single-center randomized controlled trial study was conducted in the period between June 2015 and December 2015. We obtained the approval from the Ethics Committee of National Taiwan University hospital (No. 201502043RINC), and registered the Clinical trial.gov (NCT02462915). Patients were included if they (1) had an American Society of Anesthesiologists physical status score I or II, (2) were aged 20-80 years old, (3) had an entire surgery time of less than $3 \mathrm{~h}$, and (4) had undergone an elective gynecologic laparoscopy. Patients were excluded if they exhibited lung, heart, or brain disease; pathology of the neck or upper respiratory tract; difficult intubation; increased risk of aspiration (gastroesophageal reflux or full stomach); obesity (body mass index $>30$ ) or pregnancy. After written the informed consent, the patient candidate randomized by a computer-generated random number table. Using the random number table, Patients were randomly allocated into two groups: 20 patients were treated using the i-gel and 20 patients were treated using ETTs. The surgeon were blind to our airway management and a blind observer recorded our outcome.

The patients in both groups received standardized anesthesia using the following procedure. Before anesthetic induction, the anesthetic machine and circuits were checked as the manufacturers' guidelines. We attached standard monitors to our patients, such as pulse oximeter, electrocardiography, noninvasive blood pressure. Then patients received preoxygenation for $3 \mathrm{~min}$ without bagand-mask ventilation to prevent stomach fullness. Induction was then performed using routine medication: fentanyl 1-2ug kg-1, Propofol 2-3 $\mathrm{mg} \mathrm{kg}^{-1}$ and cisatracurium $0.2 \mathrm{mg} \mathrm{kg}^{-1}$. Neuromuscular blockade was then confirmed by monitoring the train-of-four stimulation until the count achieved zero $(\mathrm{TOF}=0)$. Accordingly, either an ETT or an i-gel was inserted. For the i-gel group, the i-gel size selection depended on the patient's body weight. Size three was used for patients who weighed less than $50 \mathrm{~kg}$, size four for $50-90 \mathrm{~kg}$, and size five for those who weighed more than $90 \mathrm{~kg}$. Tracheal tube size was 7.0 for female patients. Anesthesia maintenance was obtained by end-tidal concentration of sevoflurane $2-3 \%$ in a $50 \%$ oxygen and $50 \%$ air mixture.

After the i-gel or ETT insertion, patients were ventilated using volume controlled ventilation $\left(8 \mathrm{~mL} \mathrm{~kg} \mathrm{~kg}^{-1}\right)$ of body weight with a respiratory rate of $8-16$ breaths $\mathrm{min}^{-1}$, an inspiratory-to-expiratory ratio of $1: 2$, and $40 \%$ inspired oxygen in air with a fresh flow gas rate of $3 \mathrm{~L} \mathrm{~min}^{-1}$ [18]. Sufficient ventilation was defined as a square-wave with $\mathrm{EtCO}_{2}$ values of $30-45 \mathrm{~cm} \mathrm{H}_{2} \mathrm{O}$. If some emergencies were noted in the i-gel group, such as airway obstruction or hemodynamic unstable, the i-gel was removed. Then, the patient was recorded failure, and underwent endotracheal intubation.

To collect our data, we recorded respiratory parameters when patients were in the supine phase and LPT position, which is at $25^{\circ}$ (AMSCO 3085 SP Surgical Table) and at which the intra-abdominal pressure is $12 \mathrm{mmHg}$. The mean values for $5 \mathrm{~min}$ were obtained using a GE S/5 Compact Anesthesia Monitor (GE Healthcare, Helsinki, Finland) with a spirometry tube (GE Healthcare, Helsinki, Finland) and D-lite sensor (GE Healthcare, Helsinki, Finland) (Fig. 1).

In our study, the primary outcome was the leak fraction. The leak fraction was subsequently defined as leak volume divided by inspired tidal volume (leak volume/ITV). Leak volume was defined as the difference between the inspired tidal volume (ITV) and the expired tidal volume (ETV).

Oropharyngeal leak pressure (OLP) was recorded in the supine and LPT positions using the auscultation and plateau methods [19]. The auscultation method involved placing a stethoscope at the laryngeal inlet. We squeezed the reservoir bag, until we reached the lowest pressure at which gas could be heard escaping though the stethoscope. The plateau method involved closing the pressure-limiting valve of the circuit, and adjusting the fresh gas flow to $3 \mathrm{~L} \mathrm{~min}{ }^{-1}$. We then recorded the plateau airway pressure. To avoid the possibility of gastric insufflation, the airway pressure was not allowed to exceed $30 \mathrm{~cm} \mathrm{H}_{2} \mathrm{O}$ for either method. In addition, the cuff pressure of the ETT was set to $30 \mathrm{cmH}_{2} \mathrm{O}$ using a pocket cuff pressure gauge $[20,21]$.

We suctioned the patients in the i-gel group through the gastric channel prior to extubation. Following extubation or i-gel removal we observed whether there was any vomitus in any of the patients' mouths.

In the postoperative care unit, patients were followed-up for perioperative respiratory complications and clinical signs of aspiration. They were also observed by nurse anesthetists who were blind to the purposes of the study and 


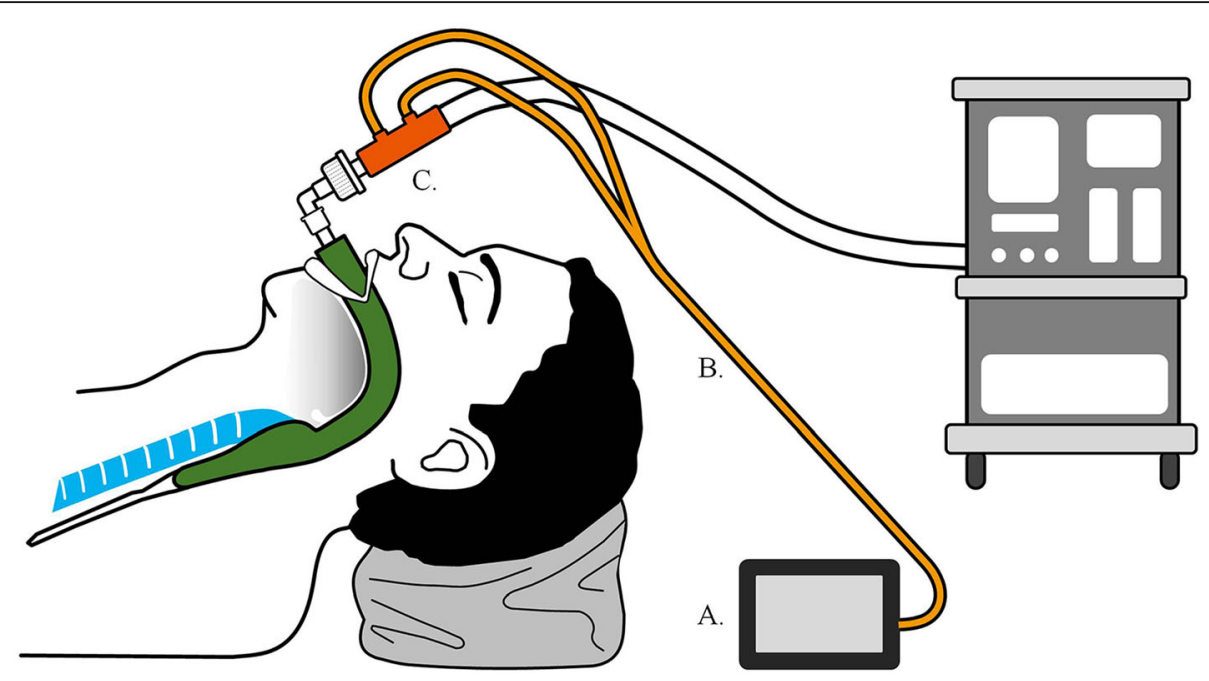

Fig. 1 Illustration of experimental settings. a GE S/5 compact anesthesia monitor (GE Healthcare, Helsinki, Finland). b spirometry tube (GE Healthcare, Helsinki, Finland). c D-lite sensor (GE Healthcare, Helsinki, Finland)

had 10 or more years' of experience for complications following the procedure. The side effects they looked for included laryngospasm, reintubation and a sore throat [22].

\section{Statistical analysis}

In this study, the primary comparison parameter was leak fraction. To estimate the sample size, based on previous research we calculated that a difference of more than 0.2 in the leak fraction between the i-gel and the ETT would be needed to be considered a significant difference [23]. There was no existing general literature on which we could base our comparison between the i-gel and the ETT in the LPT position. We also set a standard deviation value of 0.15 based on research performed by Devitt et.al [24]. We used a two-sample study design to compare group means. Analyses using MINITAB 14
Statistical Software (Minitab Inc., State College, USA) found that 10 patients were required in each group in order for our study to have $80 \%$ power and a significance level of $5 \%$.

Mann-Whitney Test were used to compare the respiratory parameter differences between i-gel and ETT groups. Wilcoxon Signed Ranks Test were used to compare the respiratory parameter difference within i-gel and ETT groups. Student's t-tests and Chi-Squared tests were used to compare the continuous and categorical data, respectively. A two-sided $P$ value of $<0.05$ was considered statistically significant. Statistical analyses were performed using SPSS 17.0 statistical software (SPSS Inc., Chicago, IL, USA).

\section{Results}

Twenty patients were enrolled into each of the i-gel and ETT groups successfully (Fig. 2). There were no

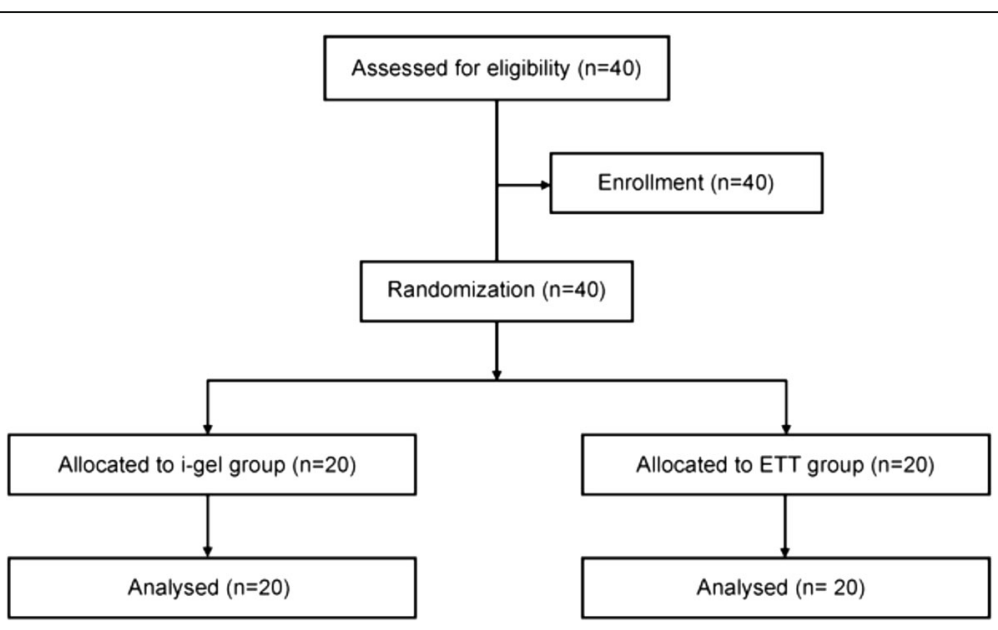

Fig. 2 Study flow diagram. ETT: endotracheal tube 
significant differences in demographic data between the two groups (Table 1). All values of the measurements (the raw data) were shown in the Additional file 1 and 2 .

For both the i-gel and ETT groups, peak and mean airway pressure and resistance were significantly higher in the LPT position than in the supine position $(p<0.001)$. Moreover, compliance was significantly lower in the LPT position than in the supine position $(p<0.001)$ (Table 2).

In the LPT position, the median [IQR] leak fractions for the i-gel and ETT groups were 6.20 [3.49] \% and $6.38[3.71] \%$, respectively $(P=0.883)$. In the supine position, the median [IQR] leak fractions for the i-gel and ETT groups were 31.99 [14.54] \% and 26.06 [9.62] \% $(P=0.341)$. In addition, there was significantly difference in the peak and mean airway pressure in the supine position for the i-gel and ETT groups (peak: 11.44 [1.43]; 12.53 [1.68], $P=0.03$, mean: 4.67 [0.71]; 4.95 [0.46], $P=0.049$ ). Furthermore, except airway pressure in supine position, there were no statistically significant differences in both positions between the groups in leak volume, compliance, or peak and mean airway pressures (Table 2). However, resistance differed between the groups for both positions, with the i-gel group exhibiting significantly lower values than those of the ETT group $(p<0.001)$.

For the i-gel group, less leakage (leak fraction: median [IQR]) was observed in the LPT position (6.20 [3.49] \%) than in the supine position (7.01 [3.73] \%) $(P=0.179)$, as illustrated in Fig. 3. For the ETT group, leakage (median [IQR]) was similar in both positions (supine : 5.76 [2.67] \%; LPT: 6.38 [3.71]\%, $P=0.194$ ) (Table 2).

\section{Postoperative recovery follow-up}

During surgery, none of the patients who received the i-gel were required to shift to intubation. In addition, vomitus was not noted in any patients following extubation or i-gel removal. In the postoperative care unit, the number of patients who reported a sore throat was significantly lower in the i-gel group (3) than in the ETT group $(9 ; p=0.038)$. No patients in either group

Table 1 Patient characteristics

\begin{tabular}{|c|c|c|c|c|}
\hline & \multirow{2}{*}{$\begin{array}{l}\text { i-gel } \\
(n=20)\end{array}$} & \multirow{2}{*}{$\begin{array}{l}\mathrm{ETT} \\
(n=20)\end{array}$} & \multirow[t]{2}{*}{$P$ value } \\
\hline & & & & \\
\hline \multicolumn{2}{|c|}{ Age (yr) } & $36.60 \pm 12.03$ & $40.65 \pm 7.26$ & 0.205 \\
\hline \multicolumn{2}{|l|}{ BMI } & $21.46 \pm 3.13$ & $21.27 \pm 2.70$ & 0.839 \\
\hline \multicolumn{2}{|c|}{ Surgery time (min) } & $111.55 \pm 43.80$ & $117.45 \pm 47.07$ & 0.684 \\
\hline \multirow[t]{2}{*}{ ASA } & (I) & $10(50 \%)$ & $13(65 \%)$ & \multirow[t]{2}{*}{0.337} \\
\hline & (II) & $10(50 \%)$ & $7(35 \%)$ & \\
\hline
\end{tabular}

ETT endotracheal tube

Data are means $\pm S D$ reported any symptoms of aspiration or following respiratory complications, including cough, laryngospasm or reintubation in the postoperative care unit. They also did not report any of the aspiration following complications prior to discharge. All patients were discharged uneventfully.

\section{Discussion}

Our findings indicate that the i-gel provides a suitable alternative to the ETT when using LPT positioning. The i-gel group produced similar leak fractions, leak volumes, and airway pressure to the ETT group. In addition, the i-gel group had significantly lower resistance and higher compliance compared with the ETT group. In addition, in the i-gel group, we observed lower leak fractions and volumes in the LPT position than in the supine position with our sample of nonobese patients. Following surgery we observed fewer cases of sore throat in the i-gel group than in the ETT group, and no clinical or endoscopic signs of aspiration in either groups.

In this study, we observed no clinical signs associated with aspiration in the i-gel group. This is because the i-gel has an esophageal channel, which enables the release of pressure induced by abdominal insufflation and head-down position during the perioperative period. In addition, the i-gel provides better sealing than first generation SADs. This means that the i-gel can completely separate the gastrointestinal and respiratory tracts and protect the airway safety, even if vomiting occurs $[10,13]$.

In the i-gel group, we observed a lower leak fraction and volume during laparoscopy when patients were in the Trendelenburg position compared with the supine position. Our finding is contrary to Carron et al. who found higher gas leakage using LMA-Proseal in laparoscopy with the reverse Trendelenburg position [25]. This suggests that the positioning change may be a major factor affecting gas leakage when using SADs.

We propose two explanations for our findings. First, the partial body weight, cephalic viscera and diaphragm pressure caused by the pneumoperitoneum and Trendelenburg position (Force B as shown in Fig. 4) may result in a tighter seal in the LPT position compared with the supine position. Alternatively, it is possible that the airway undergoes a configuration change in the LPT position, yielding a superior sealing pressure with the $\mathrm{i}$ gel [26].

In the LPT and supine positions, resistance was significantly lower in the i-gel group than in the ETT group. We propose that this result was caused by the internal diameter being larger than that of the ETT. The size four i-gel is shorter $(192 \mathrm{~mm})$ and has a larger cross section (internal diameter: $12.3 \mathrm{~mm}$ ) than the size 
Table 2 Respiratory parameter changes in supine and pneumoperitoneum and Trendelenburg position

\begin{tabular}{|c|c|c|c|c|c|c|}
\hline & \multicolumn{3}{|l|}{ Supine } & \multicolumn{3}{|c|}{ Pneumoperitoneum and Trendelenburg } \\
\hline & i-gel & ETT & $\overline{p \text {-value }}{ }^{a}$ & $\begin{array}{l}\text { i-gel } \\
4\end{array}$ & ETT & $p$-value ${ }^{b}$ \\
\hline Leak Fraction (\%) & $7.01[3.73]$ & $5.76[2.67]$ & 0.341 & $6.20[3.49]$ & $6.38[3.71]$ & 0.883 \\
\hline Leak Volume (ml) & $31.99[14.54]$ & 26.06 [9.62] & 0.327 & $27.66[1.73]$ & $28.35[18.18]$ & 0.968 \\
\hline Resistance $\left(\mathrm{cmH}_{2} \mathrm{O} / \mathrm{l} / \mathrm{s}\right)$ & $5.82[1.18]$ & $10.25[1.03]$ & $<0.01$ & $7.95[1.57]$ & $13.13[1.71]$ & $<0.01$ \\
\hline Peak Airway Pressure $\left(\mathrm{cmH}_{2} \mathrm{O}\right)$ & $11.44[1.43]$ & $12.53[1.68]$ & 0.03 & $20.75[2.75]$ & $21.60[3.24]$ & 0.461 \\
\hline Mean Airway Pressure $\left(\mathrm{cmH}_{2} \mathrm{O}\right)$ & $4.67[0.71]$ & $4.95[0.46]$ & 0.049 & $6.73[1.33]$ & $6.65[0.98]$ & 0.841 \\
\hline Compliance $\left(\mathrm{ml} / \mathrm{cmH}_{2} \mathrm{O}\right)$ & $48.45[7.98]$ & $46.34[8.13]$ & 0.62 & $23.02[4.12]$ & $23.19[4.64]$ & 0.779 \\
\hline
\end{tabular}

ETT endotracheal tube

Data are median [IQR]

${ }^{a} p$-value in comparison with i-gel and ETT in supine

${ }^{\mathrm{b}} p$-value in comparison with i-gel and ETT in Pneumoperitoneum and Trendelenburg position

7.0 ETT (length: $310 \mathrm{~mm}$; internal diameter: $7.0 \mathrm{~mm}$ ) [27]. According to the Poiseuille equation (resistance $=(8 \times$ length $\times$ viscosity $) /\left(\pi \times\right.$ radius $\left.\left.^{4}\right)\right)$ [28], the resist ance of ETT is 15.34 times greater than the i-gel. In addition, in the supine position, airway pressure in ETT was significantly higher than i-gel group. It because the diameter of i-gel is larger than ETT due to Poiseuille equation, too.

In the postoperative care unit, we observed significantly lower rates of sore throat in the i-gel group than in the ETT group. This result is similar to the findings of previous research, which compared ETTs with other disposable SADs [29]. The non-inflatable cuff of the i- gel provides low mucosal pressure [30], and can prevent hyperinflation caused by the diffusion of gases, such as nitrous oxide [31]. This results in a decreased compression of the microvascular structures and terminal nerve endings in the peri-laryngeal tissues [32].

This study has some identifiable limitations. Although we did not observe any symptoms or signs of aspiration, a larger sample size is needed to confirm the safety of the i-gel. The incidence of aspiration with the LMA Classic has been estimated at $0.02 \%$. This rate is similar to that obtained with tracheal intubation in elective patients [33]. Further investigation is also needed in patients with morbid obesity, symptoms of

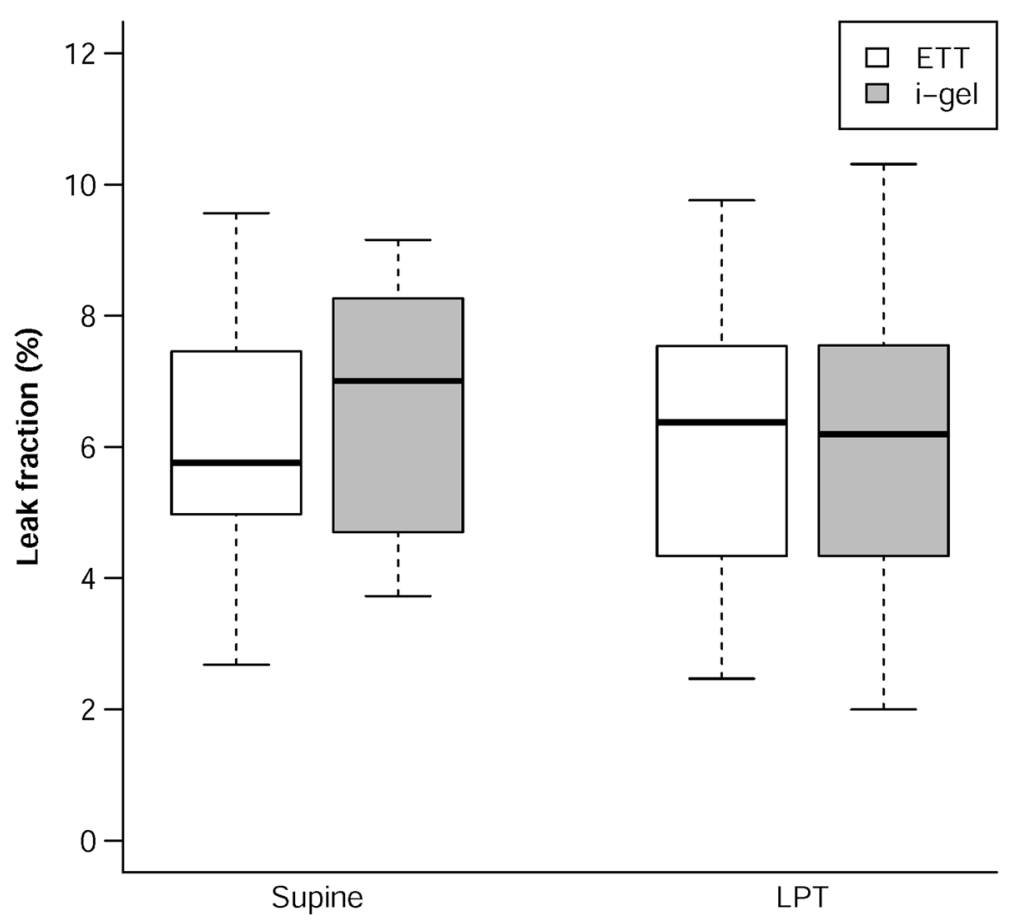

Fig. 3 Boxplot of leak fraction for i-gel and ETT groups. Data are median [IQR]. ETT: endotracheal tube 


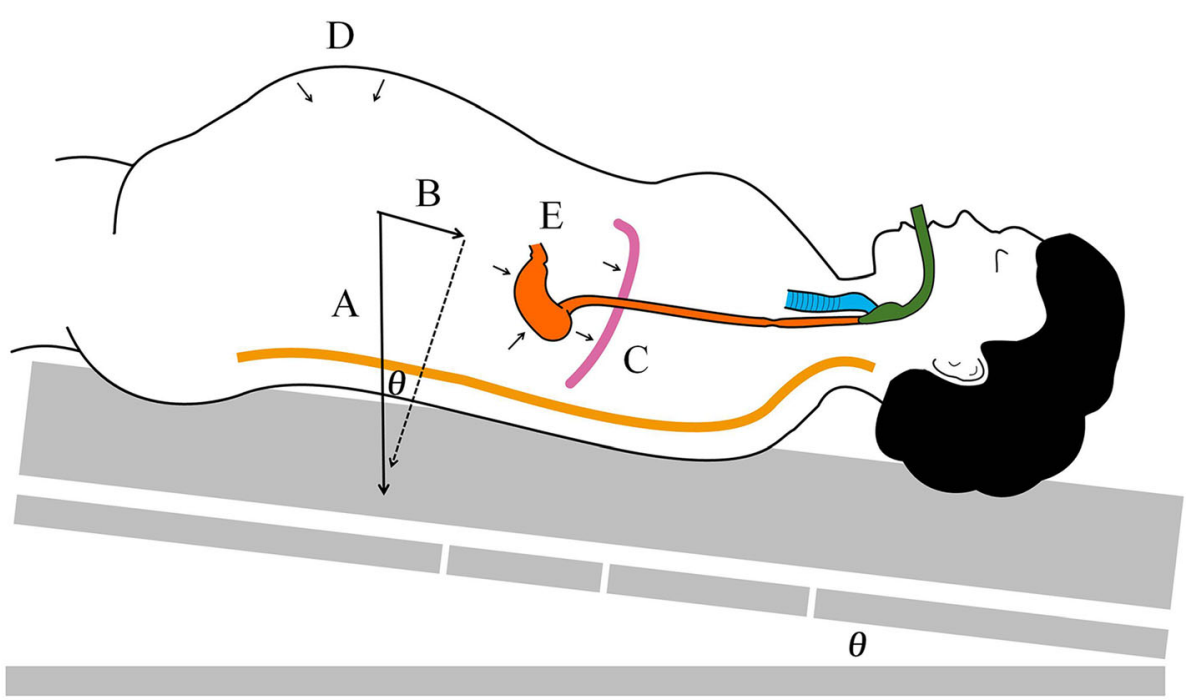

Fig. 4 The force causes less leak fraction in the LPT position than in the supine position. $a$ Body weight force. $b$ Component of body weight and cephalic shifting pressure due to LPT position. $c$ LPT position induced force on the diaphragm. $d$ Force caused by pneumoperitoneum. $e$ LPT position induced pressure on the stomach. LPT: Laparoscopic pneumoperitoneum and Trendelenburg

gastroesophageal reflux, and those undergoing operations of a longer duration. In addition, in the $\mathrm{i}$-gel group, we found that the less leakage in the LPT position was noted than in the supine position, and this phenomenon is needed further investigated in the future.

\section{Conclusions}

Our data supports that the i-gel provides a suitable alternative to the ETT for use in surgeries that use the LPT position. The i-gel provides a similar leak fraction and significantly lower resistance than the ETT. Moreover, the i-gel group displayed no evidence of aspiration, and reported lower incidences of sore throat.

\section{Additional files}

Additional file 1: "Demographic and postoperative complication changes" This file contains raw data about demographic and postoperative complications. (XLSX $13 \mathrm{~kb}$ )

Additional file 2: "Respiratory parameters" This file contains raw data about the respiratory parameters, including leak fraction, leak volume, etc. (XLSX $14 \mathrm{~kb})$

\section{Abbreviations}

ASA: American Society of Anesthesiologists; BMI: Body mass index; ETT: Endotracheal tube; LPT: Laparoscopic pneumoperitoneum and Trendelenburg

\section{Acknowledgements}

We thank the following visiting staff to help us collecting data: H.H. Huang, H.M. Yeh, and H.L. Cheng. We also thank Y.C. Yeh and Y.J Cheng for giving us the suggestion.

\section{Funding}

This work was supported without funding sources.

\section{Availability of data and materials}

The datasets supporting the conclusions of this article are included in the manuscript.

\section{Authors' contributions}

$C J L$ participated in the collection and analysis of the data and drafted the manuscript. CML and CYW participated in the design of project and drafted the manuscript. FFT and PHT participated in its design and drafted the manuscript. SZF guided the design of project and participated in the formulation of the manuscript. All authors read and approved the final manuscript.

\section{Competing interests}

The authors declare that they have no competing interests.

\section{Consent for publication}

Not applicable.

\section{Ethics approval and consent to participate}

The study was approved by the written consent of the institutional Ethical Board of the National Taiwan University Hospital, which number is 201502043RINC. All the patient consents were obtained to include any individual person's data.

\section{Author details}

${ }^{1}$ Department of Anesthesiology, National Taiwan University Hospital Hsin-Chu Branch, NO. 25, Lane 442, Sec.1, Jingguo Rd., Hsinchu City 30059, Taiwan (R.O.C.). '² Department of Anesthesiology, National Taiwan University Hospital, No. 7, Zhung Shan S. Rd., Zhongzheng Dist., Taipei City 10002, Taiwan (R.O.C.). ${ }^{3}$ Department of Internal Medicine, National Taiwan University Hospital, No. 7, Zhung Shan S. Rd., Zhongzheng Dist., Taipei City 10002, Taiwan (R.O.C.).

Received: 14 July 2016 Accepted: 6 December 2016 Published online: 06 January 2017 


\section{References}

1. Bardoczky Gl, Engelman E, Levarlet M, Simon P. Ventilatory effects of pneumoperitoneum monitored with continuous spirometry. Anaesthesia. 1993;48:309-11.

2. Ng A, Smith G. Gastroesophageal reflux and aspiration of gastric contents in anesthetic practice. Anesth Analg. 2001:93:494-513.

3. Asai T. Editorial II: Who is at increased risk of pulmonary aspiration? Br J Anaesth. 2004;93:497-500.

4. Castle N, Owen R, Hann M, Naidoo R, Reeves D. Assessment of the speed and ease of insertion of three supraglottic airway devices by paramedics: a manikin study. Emerg Med J. 2010;27:860-3.

5. Cork RC, Depa RM, Standen JR. Prospective comparison of use of the laryngeal mask and endotracheal tube for ambulatory surgery. Anes Analg. 1994;79:719-27.

6. Higgins PP, Chung F, Mezei G. Postoperative sore throat after ambulatory surgery. Br J Anaesth. 2002;88:582-4.

7. Maltby JR, Beriault MT, Watson NC, Liepert DJ, Fick GH. LMA-Classic and LMA-ProSeal are effective alternatives to endotracheal intubation for gynecologic laparoscopy. Can J Anaesth. 2003;50:71-7.

8. Abdi W, Amathieu R, Adhoum A, Poncelet C, Slavov V, Kamoun W, Combes $X$, Dhonneur G. Sparing the larynx during gynecological laparoscopy: a randomized trial comparing the LMA Supreme and the ETT. Acta Anaesthesiol Scand. 2010:54:141-6.

9. Teoh WH, Lee KM, Suhitharan T, Yahaya Z, Teo MM, Sia AT. Comparison of the LMA Supreme vs the i-gel in paralysed patients undergoing gynaecological laparoscopic surgery with controlled ventilation. Anaesthesia. 2010;65:1173-9.

10. Ali A, Canturk S, Turkmen A, Turgut N, Altan A. Comparison of the laryngeal mask airway Supreme and laryngeal mask airway Classic in adults. Eur J Anaesthesiol. 2009;26:1010-4

11. Cook TM, Lee G, Nolan JP. The ProSeal laryngeal mask airway: a review of the literature. Can J Anaesth. 2005;52:739-60.

12. de Montblanc J, Ruscio L, Mazoit JX, Benhamou D. A systematic review and meta-analysis of the i-gel((R)) vs laryngeal mask airway in adults. Anaesthesia. 2014;69:1151-62.

13. Wong DT, Yang JJ, Jagannathan N. Brief review: The LMA Supreme supraglottic airway. Can J Anaesth. 2012;59:483-93.

14. Wharton NM, Gibbison B, Gabbott DA, Haslam GM, Muchatuta N, Cook TM I-gel insertion by novices in manikins and patients. Anaesthesia. 2008;63:991-5.

15. Theiler LG, Kleine-Brueggeney M, Luepold B, Stucki F, Seiler S, Urwyler N, Greif R. Performance of the pediatric-sized i-gel compared with the Ambu AuraOnce laryngeal mask in anesthetized and ventilated children. Anesthesiology. 2011;115:102-10.

16. Levitan RM, Kinkle WC. Initial anatomic investigations of the I-gel airway: a novel supraglottic airway without inflatable cuff. Anaesthesia. 2005;60:1022-6.

17. Komasawa N, Nishihara I, Tatsumi S, Minami T. Prewarming of the i-gel facilitates successful insertion and ventilation efficacy with muscle relaxation: a randomized study. J Clin Anesth. 2014;26:663-7.

18. Kim YB, Chang YJ, Jung WS, Byen SH, Jo YY. Application of PEEP using the igel during volume-controlled ventilation in anesthetized, paralyzed patients. J Anesthesia. 2013;27:827-31.

19. Keller C, Brimacombe JR, Keller K, Morris R. Comparison of four methods for assessing airway sealing pressure with the laryngeal mask airway in adult patients. Br J Anaesth. 1999;82:286-7.

20. Tobias JD, Schwartz L, Rice J, Jatana K, Kang DR. Cuffed endotracheal tubes in infants and children: should we routinely measure the cuff pressure? Int J Pediatr Otorhinolaryngol. 2012;76:61-3.

21. Wu CY, Yeh YC, Wang MC, Lai CH, Fan SZ. Changes in endotracheal tube cuff pressure during laparoscopic surgery in head-up or head-down position. BMC Anesthesiol. 2014;14:75.

22. Kluger MT, Visvanathan T, Myburgh JA, Westhorpe RN. Crisis management during anaesthesia: regurgitation, vomiting, and aspiration. Qual Saf Health Care. 2005;14:e4

23. Uppal V, Fletcher G, Kinsella J. Comparison of the i-gel with the cuffed tracheal tube during pressure-controlled ventilation. Br J Anaesth. 2009;102:264-8.

24. Devitt JH, Wenstone R, Noel AG, O'Donnell MP. The laryngeal mask airway and positive-pressure ventilation. Anesthesiology. 1994;80:550-5.

25. Carron MDM, Veronese MDS, Gomiero PDW, Foletto MDM, Nitti MDD, Ori MDC, Freo MDU. Hemodynamic and hormonal stress responses to endotracheal tube and ProSeal laryngeal mask AirwayTMfor laparoscopic gastric banding. Anesthesiology. 2012;117:309-20.
26. Greenland KB, Edwards MJ, Hutton NJ, Challis VJ, Irwin MG, Sleigh JW. Changes in airway configuration with different head and neck positions using magnetic resonance imaging of normal airways: a new concept with possible clinical applications. Br J Anaesth. 2010;105:683-90.

27. de Lloyd L, Hodzovic I, Voisey S, Wilkes AR, Latto IP. Comparison of fibrescope guided intubation via the classic laryngeal mask airway and i-gel in a manikin. Anaesthesia. 2010;65:36-43.

28. William Henderson PAP, Najib T. AYAS. Respiratory System Mechanics and Energetics. In: Broaddus VC, editor. Nadel's Textbook of Respiratory Medicine. Canada: Saunders imprint of Elsevier; 2016. p. 85.

29. Heuer JF, Stiller M, Rathgeber J, Eich C, Züchner K, Bauer M, Timmermann A. Evaluation of the new supraglottic airway devices Ambu AuraOnce ${ }^{T M}$ and Intersurgical i-gelT. Anaesthesist. 2009;58:813-20.

30. Eschertzhuber S, Brimacombe J, Kaufmann M, Keller C, Tiefenthaler W. Directly measured mucosal pressures produced by the i-gelTM and laryngeal mask airway SupremeTM in paralysed anaesthetised patients. Anaesthesia. 2012;67:407-10.

31. Maino P, Dullenkopf A, Bernet V, Weiss M. Nitrous oxide diffusion into the cuffs of disposable laryngeal mask airways. Anaesthesia. 2005;60:278-82.

32. Park SK, Choi GJ, Choi YS, Ahn EJ, Kang H. Comparison of the i-gel and the laryngeal mask airway proseal during general anesthesia: a systematic review and meta-analysis. PLoS One. 2015;10:e0119469.

33. Brimacombe JR, Berry A. The incidence of aspiration associated with the laryngeal mask airway: A meta-analysis of published literature. J Clin Anesth. 1995; 7:297-305

\section{Submit your next manuscript to BioMed Central and we will help you at every step:}

- We accept pre-submission inquiries

- Our selector tool helps you to find the most relevant journal

- We provide round the clock customer support

- Convenient online submission

- Thorough peer review

- Inclusion in PubMed and all major indexing services

- Maximum visibility for your research

Submit your manuscript at www.biomedcentral.com/submit

) Biomed Central 